This item was submitted to Loughborough's Research Repository by the author.

Items in Figshare are protected by copyright, with all rights reserved, unless otherwise indicated.

\title{
Connectionless indoor inventory tracking in Zigbee RFID sensor network
}

PLEASE CITE THE PUBLISHED VERSION

http://dx.doi.org/10.1109/IECON.2009.5415262

PUBLISHER

(c) IEEE

VERSION

AM (Accepted Manuscript)

LICENCE

CC BY-NC-ND 4.0

REPOSITORY RECORD

Yang, Huanjia, and Shuang-Hua Yang. 2019. "Connectionless Indoor Inventory Tracking in Zigbee RFID Sensor Network". figshare. https://hdl.handle.net/2134/11876. 
This item was submitted to Loughborough's Institutional Repository (https://dspace.lboro.ac.uk/) by the author and is made available under the following Creative Commons Licence conditions.

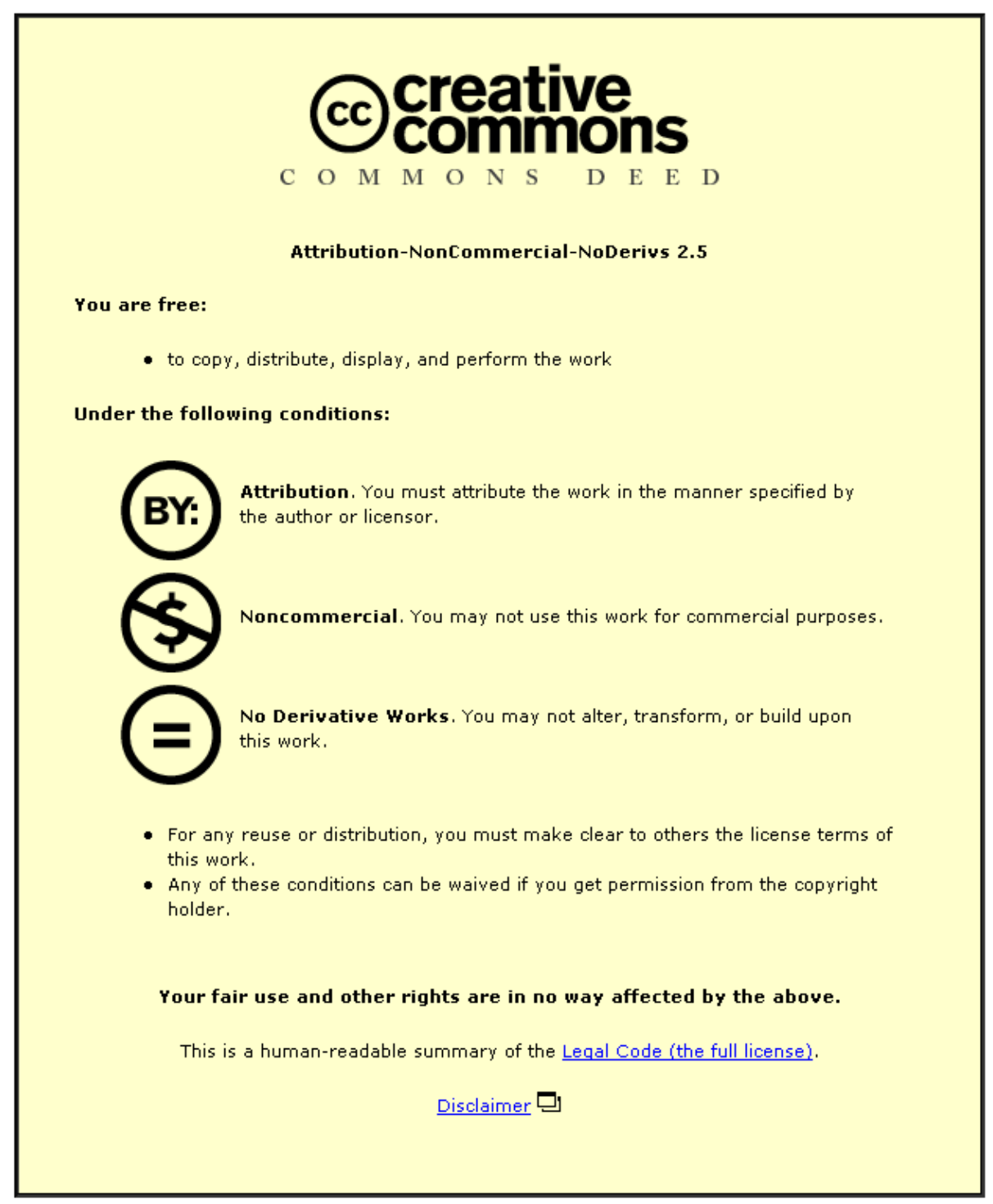

For the full text of this licence, please go to: http://creativecommons.org/licenses/by-nc-nd/2.5/ 


\title{
Connectionless Indoor Inventory Tracking in Zigbee RFID Sensor Network
}

\author{
Huanjia Yang, Shuang-Hua Yang \\ Computer Science Department, Loughborough University \\ \{H.Yang, S.H.Yang\}@lboro.ac.uk
}

\begin{abstract}
Zigbee is one of the most exciting wireless sensor network (WSN) technologies for monitoring and control. In our previous research, an integrated Zigbee RFID sensor network was designed as an 'all-in-one' system solution for Humanitarian Logistics Center (HLC) resource management. Various field trials, which have justified the feasibility and features of such a system structure, have also revealed the requirement for simple yet reliable mobile tracking architecture for Zigbee network. In this paper a connectionless tracking architecture based on Zigbee RFID sensor network is proposed for inventory management applications. Such architecture features a consistent network structure, low hardware energy consumption and no accumulated error for localization algorithms with the least additional cost and hardware required on top of the existing Zigbee RFID sensor network systems. A simple demo system is also developed to demonstrate the feasibility of our design.
\end{abstract}

Keywords: Zigbee, inventory tracking, RFID sensor network

\section{INTRODUCTION}

In the general field of logistics management research, much work has been done to prove that improving the whole supply chain performance relies on improving the external service quality at each distribution point on the chain, which requires the internal service performance at each distribution point to be improved first [1]. This is particularly important in humanitarian logistics. In fact, Thomas [2] suggests that the speed of response for major humanitarian programmes depends on the ability of logisticians to procure, transport and receive supplies at the site of a humanitarian effort, such as the humanitarian logistics centers (HLCs) which are the most important sites where both freight and information flows are congregated, relayed or distributed. Systems such as typical RFID systems and information networks have been implemented in some of the logistics centers in the general supply chain, but the fast emergency response features of humanitarian logistics prevent them from being adopted directly in humanitarian logistics centers. As a result an integrated Zigbee enabled RFID sensor network architecture has been proposed in our previous research [3][4] aiming to help increase the efficiency of each humanitarian distribution point/centre by providing higher freight and resource visibility and state monitoring ability for internal process management. Such architecture integrates sensors, passive and active RFID systems into a unified Wireless Sensor Network backbone. We have demonstrated in our previous work that it can provide the distribution centers in the humanitarian supply chain with a simple, robust, fast-to- implement and multifunctional information system infrastructure. But in our previous demonstration system in [4] problems were raised regarding the architecture of current Zigbee based tracking solutions, which have dense device implementation, mobile routers in the network and low battery life for mobile nodes. In an attempt to overcome those issues, in this paper we propose a connectionless inventory tracking architecture for indoor Zigbee RFID sensor network that support warehouse inventory tracking with the least additional hardware required, reasonable mobile node battery life and at the same time provide the server localization algorithms with accurate data input by avoiding the accumulated localization error. The tracked targets in our design are mainly considered to be the pallets and trays used in warehouse for standard goods transfer and storage.

The remainder of this paper is organized in the following order: first we briefly introduce Zigbee and the integrated Zigbee RFID sensor network designed in our previous research; then current prototypes for Zigbee based mobile tracking are discussed; after that our connectionless tracking solution is presented with explanation in network architecture in addition to its implementation in a warehouse; this is followed by a simple demo system showing the feasibility of our design. The end of the paper contains a discussion and conclusion on our current design and any future work.

\section{A. Zigbee and Zigbee RFID Sensor Network}

Zigbee is a wireless communication standard proposed and maintained by the Zigbee alliance. It is built on the IEEE802.15.4 standard featuring reliable, cost-effective, lowpower, wirelessly networked monitoring and control products [5]. Because the communication components are supported by IEEE 802.15 .4 , Zigbee can provide excellent peer-to-peer communication. The upper layer-network layer defined by the Zigbee specification [6] gives Zigbee the ability to extend the range of the network. There are two basic and one advanced network topologies supported: star, tree and mesh. Known as a sensor network standard Zigbee can have up to 65535 nodes in any one network, with a self organized and self healing network structure it provides a multi-hop data relaying within the network for communication between any two nodes.

In our previous research an integrated hybrid RFID sensor network system architecture was designed as an all-in-one system solution for HLC management, which is shown in figure 1 [4]. The application of the architecture was also extendable to fit a general warehouses. 


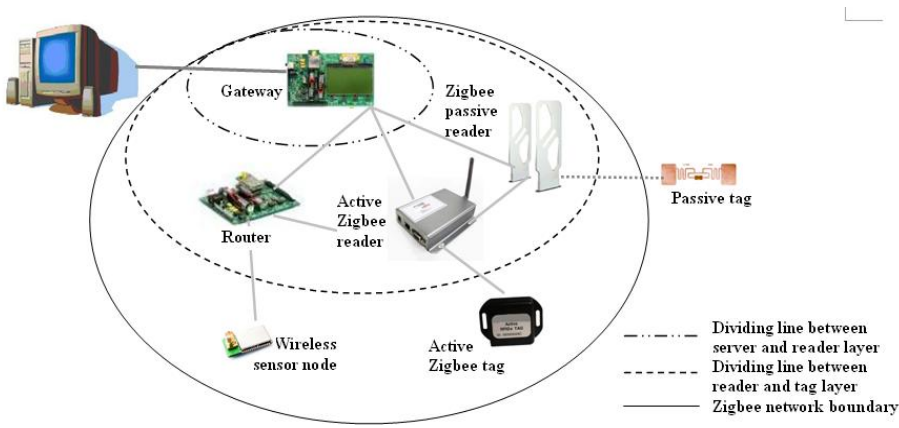

Figure 1. Integrated RFID sensor network system architecture [4]

In this integrated architecture all communications inside the network are supported by Zigbee except between the passive RFID tags and readers. Zigbee routers are modified to be able to read mobile wireless sensor nodes ID like active RFID system. Mobile readers, no matter passive or virtual active readers, can benefit from WSN protocol to connect with the server through the other readers and router devices using multi-hop communication. Dedicated wireless sensor nodes without an ID function can be implemented in the scenario as a supplement for environment monitoring, which is a typical task for a pure Wireless Sensor Networks. The flexibility of the sensor network architecture allows modularization design in system development. Sensor nodes, active and passive RFID readers can be transformed into system compatible, plug and play modules. This can simplify the design and implementation of the final system for various Warehouses.

\section{B. Current Prototypes for Zigbee-based Tracking}

One of the main tasks of the Zigbee RFID sensor network is inventory tracking. Various solutions have been proposed by researchers for Zigbee based mobile tracking. The authors in [7] proposed a Zigbee indoor tracking system in which all the reference nodes and mobile target nodes are Zigbee router devices. This ensured a fully connected network in which the mobile node can communicate with all the reference points nearby in order to satisfy the centroid localization algorithm adopted. The prototype proposed in [8] used similar but improved network architecture by modifying the localization algorithm with a triangle algorithm and weighted LQI model. Such systems require dense router deployment and the whole network is a full router network. But in most cases, this is not very practical for real applications. Typical Zigbee network have only a small number of router devices in the network while most of the task nodes are end devices so that they can be kept in sleeping mode most of the time to save battery life.

The authors in [9] proposed an improved model in which the fixed reference points can be either Zigbee router or end device. Mobile nodes are still routers and using a proposed re-connection phase the mobile router can get access to all the nearby fixed nodes. The mechanism in the re-connection phase is actually forcing the network to re-organize and hope that the nearby fixed end devices can change their parent node to the mobile router. The problem with such a system is that the network is under reorganization all of the time and, from our experiments with Zigbee hardware, this is a very battery consuming procedure for the end devices. Furthermore, the system performance is very likely to decrease rapidly when the number of mobile nodes in the same area increases, because when one mobile node is measuring the RF strength by communicating with the reference nodes within the area, it will fully occupy all the end device reference nodes, making them unable to talk to any other mobile nodes in the same area. Another important issue is that as all the mobile nodes in such system are Zigbee routers the system's network topology becomes inconsistent. This effect will be discussed in the next section.

A further improved model based on the system in [9] is an attempt to overcome the reference nodes occupying problem. The method proposed is when the first mobile node is being tracked and occupies some of the end device reference nodes within the area; the other mobile nodes will consider the tracked mobile node as a reference node when looking for reference triangulation. As soon as it find its own reference triangulation and is tracked, the other mobile nodes will also consider it as a reference point. While this method seems to allow multiple mobile nodes in the same area to be tracked simultaneously, its network structure is still inconsistent and it brings a new problem of accumulated localization error. Using a mobile node as a reference point will result in its localization error being partly accumulated into the error of the second mobile node's location calculation.

\section{CONNECTIONLESS TRACKING ARCHITECTURE}

Zigbee routers participate in packet relaying in the network which means they conduct frequent RF transmission and need to be active all the time. This significantly cuts down the battery life and makes the use of Zigbee routers as mobile nodes impractical in real applications. According to our experiment a Zigbee router with two AAA size batteries can work for only up to 24 hours. On the other hand, Zigbee End Devices (ZED) are based on IEEE802.15.4 RFD (Reduced Function Device); This means they have a simpler structure, cheaper cost and less energy consumption because of their simple role in the network: they are not involved in the network routing mechanism and can thus be put into sleep mode while idle. Using the same set of two AAA batteries the measurement and calculation on our hardware suggest that with a 0.5 second RF active time and 3 minute sleep time a ZED can expect a battery life up to a year, which can be further extended to 2 years by having either longer sleep time or larger batteries. This enables the mobile node to have a much reasonable battery life, thus makes ZEDs more suitable to be used as the RFID sensor tags on pallets and trays.

Another reason to favor ZED for mobile nodes is that the mobility of routers causes extreme routing overhead because their movement causes continuous changing of the logical topology in the network backbone and results in the mesh network constantly being reorganized [10]. The movements of a router also results in all the ZEDs coupled with it being disconnected and having to search and join the network again 
and again which is a very power consuming operation. The movement of router devices in Zigbee network causes heavier traffic, inconsistent network topology and larger energy consumption not only by itself but also by all the end devices.

On the other side, ZEDs are also preferred as the fixed data nodes. Firstly, they are more power efficient and could stand longer during possible power losing time in HLCs; Secondly, increasing router number results in more hierarchies in network topology, according to [11] the more hierarchy a Zigbee network has, the more complexity it has with a concomitant lowering of efficiency in mobility support.

But using ZED as mobile nodes is not a simple adoption. Tagging the freight pallets with ZEDs leads to network link number restriction problem which is the reason why the current Zigbee based tracking prototypes use router as mobile nodes. Zigbee specification limits a ZED to have only 1 upbound connection to its parent node which must be a router (ZR). This means if a mobile target is tagged with ZED it can only have one connection at the same time while a typical localization algorithm requires at least three. To overcome this problem our connectionless architecture is proposed.

\section{A. Connectionless Tracking Architecture}

People naturally think that if a mobile node needs to be tracked it has to connect to at least three reference nodes. This is the approach that all the current systems take. But actually it can be enough for the mobile nodes to simply "listen" to the reference nodes instead of having to "communicate" with them, which means they do not necessarily have to be network linked. For network standards such as Zigbee, Wi-Fi and Bluetooth, the reason why two nodes need to be network linked is that they need to have a guaranteed direct data communication. A tracked mobile node doesn't need to send data to the fixed reference nodes; and as RF power strength is measured by the receiver, it doesn't need to receive any data except for the identity of the reference nodes. Based on this idea, a connectionless tracking architecture at the network level is proposed for Zigbee RFID Sensor Network inventory management system, which is shown in Figure 2.

Network level 1: A Zigbee coordinator together with the local server at the top level of the network is responsible for the establishing and initialization of the indoor Zigbee network. The coordinator also acts as the sink node of the Zigbee network from which the server retrieves all the information gathered from the network nodes.

Network level 2: At the mid-level routers are responsible for data relaying and ensuring the coverage of the network within the building. Zigbee is a multi-hop network in which information from a terminal node goes through a virtual path constructed by a chain of routers before reaching the destination. Routers have main power so that they can always be active and can provide full network coverage as long as at least one router is reachable at anywhere in the building. Instead of the much denser router deployment required in the traditional tracking systems, this minimum requirement for network coverage is enough for our system implementation.

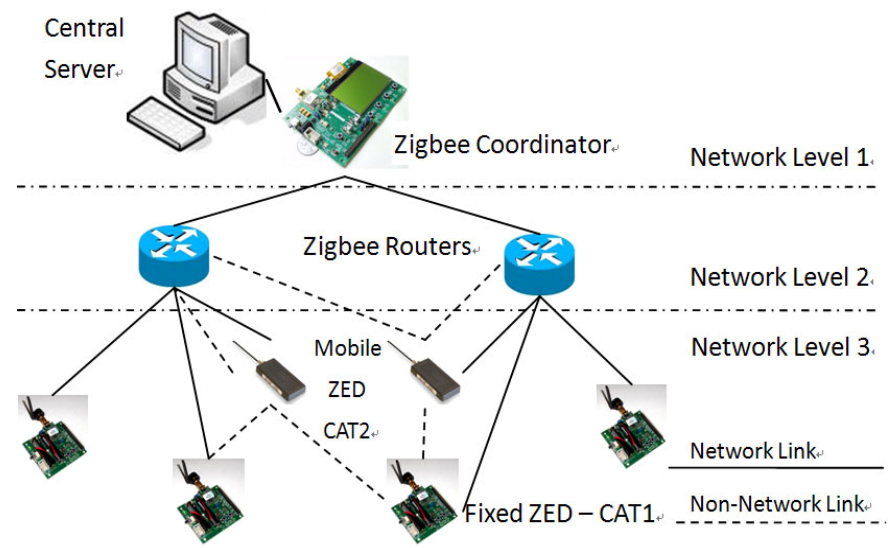

Figure 2. Connectionless tracking architecture in Zigbee

Network level 3: At the lowest level there are ZEDs for data collection. These ZEDs are divided in two categories. CAT1 includes those fixed data nodes responsible for gathering information at specific location. ZEDs carrying temperature, humidity or chemical sensors at various environment control points in the warehouse fall into this category. CAT2 includes the mobile ZEDs located on pallets or trays. The CAT1 ZEDs also act as reference nodes in the connectionless tracking system; while the other ZEDs in CAT2 make use of them as well as the routers as reference points for their localization. Dedicated reference devices, which are either Zigbee router or CAT1 node, may also be used in case where a CAT2 mobile node cannot get within the RF range of at least 3 reference nodes. CAT2 nodes also carry a passive RFID tag to be used during the goods receiving and shipping procedure for easy association and dissociation between the node and the goods on the pallet to which it is attached. We also need to consider Zigbee enabled RFID passive readers and determine their category: A reader installed at a fixed position is defined as being a CAT1 node, otherwise if it is a mobile reader carried by staff or forklift, it is defined as CAT2 node.

Data communication: The data communication of the inventory management system network is handled completely by the Zigbee standard. Primary data communications occurred in the network of our system are regular information reports from all the level 3 nodes to the coordinator/server, the data stream from the Zigbee enabled passive RFID readers at various access points to the server and the data inquiries started by the server to one or part of the network nodes. These data communications are typical point-to-point network data transmissions that can be managed by the standard network protocols used by Zigbee.

Tracking CAT2 mobile nodes: The connectionless tracking mechanism of our Zigbee RFID Sensor network can be described as follows: the CAT2 mobile nodes are typical Zigbee end devices equipped with a RF listener module whose function is to analyze the packets it can heard within the channel of the Zigbee network that the system is using. From each packet it hears the RF listener module retrieves and provides the CAT2 node processor with the ID of the reference node who sent the packet, the RF power strength and error check result. Failure of its check code indicates that 
there were collisions or significant interference during the packet transmission, this will invalid the reading of the source ID, which may have been incorrectly transmitted, and its RF power strength reading, which may be incorrect due to collision or interference. If the check passed then the source ID and RF strength reading is accepted as a reference pair, which will then be sent via the Zigbee network to the server to update the database. Proper localization algorithm on the server will locate the CAT2 nodes based on the reference information pairs in the database, while inventory tracking system then tracks goods in the warehouse by finding the corresponding inventory associated with the CAT2 node ID in the inventory database. Most of the current localization algorithms require at least three reference points for a mobile node to be located with satisfied accuracy, so the minimum implementation requirement of the connectionless tracking architecture is to ensure that at any place in the building/site, a CAT2 mobile nodes should be within the RF range of at least three reference nodes of which only one node is required to be a router providing network access. Dedicated CAT1 reference nodes can be deployed where necessary to help meeting this requirement.

\section{B. Implementation of System Network Architecture}

An illustration of the network implementation of a Zigbee enabled RFID sensor network with connectionless tracking architecture is shown in figure 3. The central server together with the Zigbee network coordinator at network level 1 can be installed in the warehouse office. The coordinator is responsible for the establishment of the Zigbee network, and acts as the network sink node from which the server will retrieve all the information collected by the network devices. The central server is expected to run the database service and exchange information with user applications on demand, thus it should be at least a dedicated PC level device with a mains power resource. As the Zigbee coordinator is physically connected with the server via a serial interface, it can easily obtain mains power supply and thus always be kept active.

Zigbee routers at network level 2 will then be deployed in the scenario. We will configure the future Zigbee network as a mesh network to enable better mobility support [10], thus the deployment criteria for the router devices are: 1). The Zigbee coordinator must be able to connect to at least one Zigbee router; 2). Each router must be able to connect to at least one other router that is reachable by the Zigbee coordinator device through a multi-hop path. The procedure for achieving a proper router deployment is like drawing a topology graph, in which the nodes are the Zigbee routers and two nodes are considered to be linked if the routers they presented are within each other's RF range. The deployment procedure can be simply described as: i).Deploy the router devices from close to the coordinator, and extend the network coverage by deploying more routers until the whole building/site is fully covered; ii). For each new router deployed, make sure it can either connect directly to the coordinator device, or connect to at least one other router that

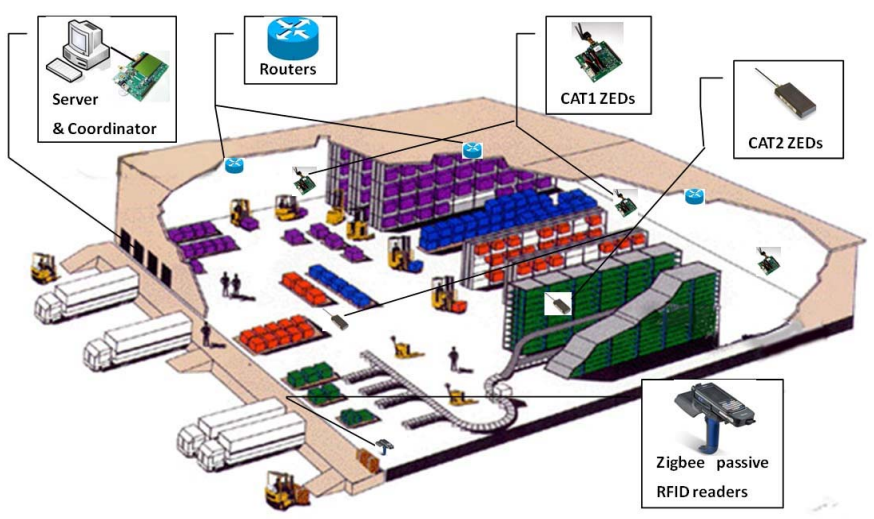

Figure 3. Zigbee Connectionless Tracking System Implementation is already deployed. Zigbee routers are supposed to be supported by mains power and always be kept active to guarantee the network connectivity. They can be deployed at locations convenient for connection to mains power as long as the deployment procedure described above is satisfied.

With full Zigbee network coverage, ZEDs in the network level 3 is then deployed. CAT1 nodes are fixed data nodes gathering information at specific locations. Their deployment will be based on the warehouse management regulations. CAT1 nodes carrying temperature sensor deployed at various temperature control points could be one such example. CAT2 nodes are deployed on the standard pallets or trays. These nodes also carry passive RFID tag so that they can be easily associated or dissociated with the inventory they are attached to by the Zigbee enabled passive RFID readers.

Since the routers and the CAT1 nodes have fixed position after deployment, they will be used as reference points for tracking CAT2 nodes. To ensure the operation of the system's tracking mechanism, dedicated CAT1 reference nodes can also be deployed to positions where a CAT2 node cannot be within the RF range of at least three reference nodes.

\section{Implementation of General Functions}

Zigbee RFID sensor network acts as the data collection network in a typical logistics management system. Functions such as layout management, location reservation, consolation and pick-to-clear concern only the data exchange between database service and user application, and thus don't require instant action of the data collection network. In our research we are only interested in the data collection network part. The functions of data collection network can be summarized as updating the inventory and environmental information in the central server database either on demand or on a regular basis, which can be based on a predefined time interval, the occurrence of certain event triggers or the mix of the two. Inventory inbound and outbound

The inventory inbound procedure is assisted mainly by Zigbee passive RFID readers at the warehouse receiving area (CAT1) or carried by staff or forklift (CAT2). The purpose of this procedure is to identify the incoming goods and allocate CAT2 node to the goods' pallet or tray. Passive RFID tags on CAT2 nodes will be read and recorded by the passive RFID reader antenna. If the incoming goods have passive tag in the 
package, they can easily be associated with the CAT2 node allocated to them through the reader. In the situation where incoming goods do not have pre-attached passive RFID tag the warehouse staff will provide the server with goods information using the input interface on the Zigbee enabled passive RFID reader [3]. Outbound/shipment of goods has very similar procedure except that the server will dissociate the goods with the CAT2 node instead of associating them.

\section{Regular environment and inventory report}

Inventory information is updated regularly to provide a relatively constant view of the goods location and condition. CAT1 nodes report goods condition to the server periodically with a predefined time interval $t$. Their communication with the server results in packet exchanging between these CAT1 and their parent nodes as well as between the routers nodes themselves. CAT2 nodes analyze this communication within its RF range for a period of $t_{1}$, identify the sender and the RF strength of packets it "heard" to generate the reference information pairs. These pairs are then sent to the server via network and are stored in the server database for localization. The CAT2 nodes will then switch to sleep mode and repeat this procedure periodically with a predefined time interval $\mathrm{T}$.

\section{Selecting the value of $t, T$ and $t_{l}$}

The power resource used by level 2, CAT1 and CAT2 nodes are different. CAT2 nodes use on board battery as power resource and thus need to have a power efficient operation strategy. Our recommendation for the value of the length of channel listening period is $t_{1} \in(t, 2 t]$. A $t_{1}$ in this range should be long enough for the CAT2 node to correctly receive packets from all the reference nodes covering it while on the other hand, not unnecessarily long in order to save the on board power. In the remainder of our discussion we set $\mathrm{t}_{\mathrm{l}}=2 \mathrm{t}$ unless otherwise noted.

The selection of the value of $\mathrm{T}$ and $\mathrm{t}$ is discussed together as they are related. After a battery is chosen and the battery capacity fixed, the power consumption of a node depends on its average working current. For Zigbee end devices like the CAT1 and CAT2 nodes, the result is also related to the length of active time and sleep time. There are two situations possible in the real applications that result in two different strategies to determine $\mathrm{t}$ and $\mathrm{T}$.

1). The first situation is that all the fixed ZED reference nodes use mains power. In this case we are only concerned about the battery life of CAT2 nodes, which can be expressed in equation 1 : $\mathrm{B}_{\mathrm{CAT} 2}$ represents the CAT2 nodes' battery life in hour, $E_{\text {battery }}$ is the capacity of the battery set carried on board in $\mathrm{mAh}, \mathrm{A}_{\mathrm{ZED}}$ is the average working current of CAT2 nodes in $\mathrm{mA}$.

$$
B_{C A T 2}=\frac{E_{\text {battery }}}{A_{\text {ZED }}} \times \frac{T}{t_{l}}=\frac{E_{\text {battery }}}{A_{\text {ZED }}} \times \frac{T}{2 t}
$$

From equation 1 , as $\mathrm{E}_{\text {battery }}$ and $\mathrm{A}_{\mathrm{ZED}}$ are decided by hardware design, they can be considered as constants in our discussion. Thus the battery life $\mathrm{B}_{\mathrm{CAT} 2}$ could be extended by simply increasing the ratio between $\mathrm{T}$ and $\mathrm{t}$. Based on this result value of $\mathrm{T}$ and $\mathrm{t}$ can be selected taking into account consideration of management regulations and requirements. For example, in a final system design $\mathrm{E}_{\text {battery }}$ and $\mathrm{A}_{\mathrm{ZED}}$ have been fixed by hardware design, and the management regulations require a battery life of $\mathrm{B}_{\operatorname{minCAT} 2}$, minimum update interval for environment monitoring at $t_{\min }$ and minimum inventory tracking update interval at $T_{\min }$. To satisfy the regulations we can use $t_{\min }$ and equation 2 to calculate a corresponding $\mathrm{T}_{\mathrm{t} \text { min }}$, and then with $\mathrm{T}_{\min }$ and equation 3 we get a corresponding $\mathrm{t}_{\mathrm{T}_{-} \min }$ :

$$
\begin{aligned}
& T_{t_{-} \min }=\frac{2 t_{\min } \times A_{Z E D} \times B_{C A T 2}}{E_{\text {battery }}} \\
& t_{T_{-} \min }=\frac{E_{\text {battery }}}{A_{\text {ZED }}} \times \frac{T_{\text {min }}}{2 B_{C A T 2}}
\end{aligned}
$$

The value of $\mathrm{T}$ and $\mathrm{t}$ could then be decided by selecting the shortest $\mathrm{T}$ or $\mathrm{t}$ that satisfies both equations.

2). the second situation is that all the end devices, which include both CAT1 and CAT2 nodes, are battery powered. In this situation we are concerned not only about the mobile CAT2 nodes, but also about the CAT1 reference nodes' battery life. This can be expressed in equation 4, in which $\mathrm{B}_{\mathrm{CAT} 1}$ represents the CAT1 nodes' battery life in hour and a new parameter $\tau$ represents the active report time of CAT1 reference nodes in each updating period t.

$$
B_{C A T 1}=\frac{E_{\text {battery }}}{A_{\text {ZED }}} \times \frac{t}{\tau}
$$

In this situation we consider the concept of network battery life, which is the time when the first out-of-battery node appears in the network: $\quad \mathbf{B}_{\text {network }}=\min \left(\mathbf{B}_{\mathbf{C A T 1}}=\mathbf{B}_{\mathrm{CAT2} 2}\right)$

To find the rule for achieving maximum value of $B_{\text {network }}$, we normalize $\mathrm{T}$ and $\mathrm{t}$ into expressions of $\tau$. For CAT1 nodes the communication time $\tau<<t<T$, let: $T=a \tau, t=b \tau$

Equation 1 and 4 are normalized as:

$$
B_{C A T 1}=b \times \frac{E_{\text {battery }}}{A_{Z E D}}, B_{C A T 2}=\frac{a}{2 b} \times \frac{E_{\text {battery }}}{A_{Z E D}}
$$

Equation (7) shows that when a $T=a \tau$ is selected, changing the coefficient $b$ of CAT1 data updating interval will affect the battery life of both CAT1 and CAT2 nodes, with inverse proportion between $\mathrm{B}_{\mathrm{CAT} 1}$ and $\mathrm{B}_{\mathrm{CAT2}}$. Hence $\mathrm{B}_{\text {network }}$ reaches its maximum value if and only if: $\quad \mathbf{B}_{\mathbf{C A T} 1}=\mathbf{B}_{\mathbf{C A T} 2}$

$$
\text { (7)(8) } \Rightarrow b \times \frac{E_{\text {battery }}}{A_{Z E D}}=\frac{a}{2 b} \times \frac{E_{\text {battery }}}{A_{Z E D}} \Rightarrow a=2 b^{2}
$$

This suggests that for a selected coefficient a (or b), $B_{\text {network }}$ reaches its maximum value if and only if $b$ (or a) satisfies equation 9. Substitute (9) back into (6), we get the ratio between $\mathrm{T}$ and $\mathrm{t}$ for achieving maximum $\mathrm{B}_{\text {network }}$.

$$
\left.\begin{array}{c}
t=b \tau \\
T=a \tau=2 b^{2} \tau
\end{array}\right\} \Rightarrow \frac{T}{t}=2 b
$$

Inventory inquiry and picking

Zigbee standard does not support remote network wake up. There is no way to activate an end device in sleep mode until it reaches the end of the preselected sleep time. Thus inquiries from user application is served by the server database service thus won't involve the data collection network. Picking procedure is assisted by the Zigbee enabled mobile passive RFID readers carried by staff or forklifts (readers in CAT2). 


\section{DEMONSTRATION SYSTEM}

We developed a simple demonstration system using the Jennic JN5139 Zigbee sensor network development kit. One module was set as the coordinator, which was used to receive messages from the remote nodes. Three more modules integrated with temperature and humidity sensors were deployed at fixed positions in our laboratory which is an open environment. These three fixed nodes consisted of one router and two ZEDs. They are considered as the environmental control points in warehouse. The last ZED was designed and programmed to be able to listen to the RF traffic and act as an end device in the network. We don't need any additional hardware to perform this function as all the nodes in a Zigbee network are all actually listening to the channel their network is using. All the packets in the channel are demodulated at the physical layer regardless of whether the packet is for the node or not. It is at the MAC layer where the packets with a destination other than the particular node are filtered. This node was considered as one of the mobile nodes on a pallet.

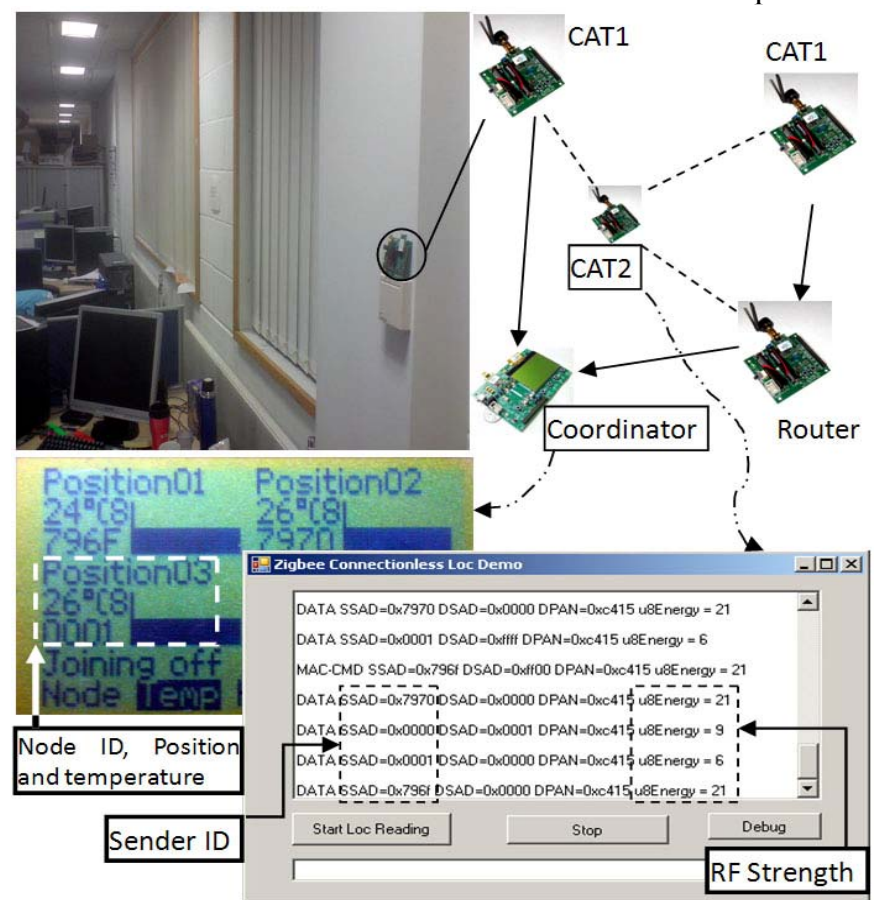

Figure 4. Zigbee Connectionless Tracking Demonstration System

Figure 4 shows the system deployment of this experiment. The coordinator initializes the network, followed by the join of the fixed router and the end devices making the network a typical Zigbee monitoring system. The fixed nodes exchange the environment information with the coordinator and this information is displayed on the screen of the coordinator's monitor. The mobile end device successfully retrieved from these data traffic the Id of the fixed nodes and their RF signal strength at its current position; this information could then be used by proper localization algorithms such as centroid, triangulation or RF finger prints. The network topology is maintained only with one normal end device joined per mobile target. This demonstration system shows the feasibility of our architecture and its hardware realization.

\section{DISCUSSION AND CONCLUSION}

The connectionless tracking architecture for Zigbee RFID sensor network allows mobile node to carry Zigbee end devices that can be supported by simpler, cheaper and power efficient hardware comparing to the router devices used in current Zigbee tracking systems. This enables the mobile node to have a much reasonable battery life subjects to the value chosen for $\mathrm{T}$ and $\mathrm{t}$.

Current Zigbee based tracking systems either requires a dense router implementation that leads to higher cost, less flexibility and more complicated network structure, or suffer accumulated localization error due to using mobile nodes as part of the reference points. The connectionless tracking architecture does not require dense router deployment. Instead, it is mainly based on the existing Zigbee RFID sensor network hardware and does not affect the network structure, implementation and performance. The data collection network could thus support warehouse inventory tracking with the least additional hardware and cost while at the same time avoid the accumulated localization error.

Challenge also exists in future research as the current architecture is not perfect for tracking high mobility target, which requires a much higher location update rate. It can efficiently carry out the tracking of inventory, which stays at a fixed position for most of the time and does not move together. By simply setting smaller value for $\mathrm{T}$ and $\mathrm{t}$ a higher update rate can be achieved in theory, but considering the nodes in network level 3 (includes CAT1 and CAT2) of our architecture are all battery assisted it is very unlikely to be practical because this will significantly reduce the battery life of both the mobile nodes and the battery assisted reference nodes. The tracking of forklift and human will require more hardware and more complicated mechanism, which is currently being investigated in our research.

\section{REFERENCES}

[1]. Conduit, J. et al, How critical is internal customer orientation to market orientation, Journal of Business Research, Vol. 51, Jan, pp. 11-24, 2001

[2]. Thomas, A., Why logistics, Forced Migration Review, Vol.18, p.4, 2003

[3]. Yang, H. et al , Zigbee Enabled RFID System, Proceedings of the IASTED International Conference on Communication Systems, Networks and Applications, pp. 163-168, Beijing, 8-10 Oct, 2007

[4]. Yang, H. et al, Hybrid RFID Sensor Network for Humanitarian Logistics Centre Management, submitted International Journal of Logistics Management

[5]. Zigbee Alliance, http://www.zigbee.org/en/about/index.asp

[6]. Zigbee Specification v1.0, Zigbee Alliance, www.zigbee.org/, 2005

[7]. Blumenthal, J. et al, Weighted centroid localization in Zigbee-based sensor networks, Folien IEEE International Symposium on Intelligent Signal Processing, WISP, Madrid, Spain, 2007

[8]. Alhmiedat, T., Yang, S., A Zigbee-based Mobile Tracking System through Wireless Sensor Networks, International Journal of Advanced Mechatronic Systems, Volume 1, Number 1, pp. 63-70, 2008

[9]. Alhmiedat, T., Yang, S., A Decentralised Low-power Zigbee-based Localization Approach, Proceedings of the 14th Internation Conference on Automation \& Computing, London, UK, September 2008

[10]. Sun, T., et al., Evaluating Mobility Support in Zigbee Networks, IFIP International Conference on Embedded and Ubiquitous Computing, Southampton, UK, 2007

[11]. Liang, N., et al., Impact of Node Heterogeneity in Zigbee Mesh Network Routing, , IEEE International Conference on Systems, Man and Cybernetics (SMC06), vol.1, pp.187-191, 8-11 Oct. 2006 